\title{
A Common Seizure Mimic Masquerading as Recurrent Status Epilepticus
}

\author{
Ranjith Kumar Manokaran ${ }^{1}$ (D) $\cdot$ R Varsha ${ }^{2}$
}

Received: 5 April 2021 / Accepted: 30 April 2021 / Published online: 20 May 2021

(C) Dr. K C Chaudhuri Foundation 2021

To the Editor: Sandifer syndrome is primarily a pediatric gastrointestinal disorder characterized by severe gastroesophageal reflux presenting with a myriad of neurological symptoms of retrocollis, dystonia, irritability, and abnormal posturing mimicking seizure [1].

A four-and-half month old, well thriving, exclusively breast-fed infant presented to us with recurrent, unexplained episodes of irritability and abnormal posturing. This child was admitted and evaluated extensively twice at an outside tertiary care hospital for similar yet exaggerated episodes following diphtheria-pertussis-tetanus (DPT) vaccination. These incidents were presumed to be status epilepticus and suspected adverse events following immunization. The child was intubated and loaded with antiepileptic medications for neuroprotection. Electroencephalography and MRI were normal, not supporting the diagnosis of epilepsy. Inborn errors of metabolism were ruled out by metabolic testing. Neurologically, the child was appropriate for age except for a partial head lag.

Ranjith Kumar Manokaran

doct.ranjith@gmail.com

1 Division of Pediatric Neurology, Department of Neurology, Sri Ramachandra Institute of Higher Education and Research, Porur, Chennai, Tamil Nadu 600116, India

2 Department of Pediatrics, Sri Ramachandra Institute of Higher Education and Research, Porur, Chennai, Tamil Nadu, India
A peculiar history was noted in the form of increase in these occurrences following feeds. This striking feature led us to consider reflux as a plausible etiology. A barium swallow study revealed a grade III gastroesophageal reflux clinching the diagnosis of Sandifer syndrome. The symptoms gradually ceased with only conservative management.

A well-known fact is Sandifer syndrome commonly mimicking a seizure [2]. This case presents an uncommon presentation of Sandifer syndrome masquerading as recurrent status epilepticus to the extent of requiring intubation and mechanical ventilation. This case highlights the significance of a thorough history and meticulous examination, which can aid us in reaching a diagnosis of uncommon presentations of a common entity so as to avoid unnecessary interventions.

\section{Declarations}

Conflict of Interest None.

\section{References}

1. Mindlina I. Diagnosis and management of Sandifer syndrome in children with intractable neurological symptoms. Eur J Pediatr. 2020;179:243-50.

2. Kabakuş N, Kurt A. Sandifer syndrome: a continuing problem of misdiagnosis. Pediatr Int. 2006;48:622-5.

Publisher's Note Springer Nature remains neutral with regard to jurisdictional claims in published maps and institutional affiliations. 